\title{
ANALYTICAL APPROACH OF SLENDER STRUCTURE VIBRATION DUE TO RANDOM COMPONENT OF THE WIND VELOCITY
}

\author{
Jiří Náprstek ${ }^{1}$, Stanislav Hračov $^{2}$ \\ ${ }^{1}$ Institute of Theoretical and Applied Mechanics \\ Prosecká 76, CZ-190 00 Prague 9, Czech Republic \\ e-mail: naprstek@itam.cas.cz \\ ${ }^{2}$ Institute of Theoretical and Applied Mechanics \\ Prosecká 76, CZ-190 00 Prague 9, Czech Republic \\ e-mail: hracov@itam.cas.cz
}

Keywords: Random vibrations, Slender structures, Wind load, Non-proportional damping, Distributed-Parameter Systems.

\begin{abstract}
Along wind random vibration of slender structures represents one of the most important aeroelastic effects resulting from wind - structure interaction. The theoretical model being based on one-dimensional elements with continuously distributed mass and stiffness has been introduced in this paper. The system has been considered to be linear self-adjoint with strongly non-proportional linear damping due to both material of the structure and presence of vibration dampers. The additive random excitation continuously distributed in time and space is Gaussian, therefore the response is Gaussian as well. Consequently, mathematical mean value and correlation function are satisfactory for the full description of the generalized solution of the respective PDE in the stochastic meaning. The general results have been obtained mostly in the form of analytical formulae for important cases of input spectral densities. A numerical example dealing with real structure is presented.
\end{abstract}




\section{INTRODUCTION}

Along wind response of tall slender structures, e.g. telecommunication towers, stacks, chimneys plays a significant role in their design stage. The wind load very often induces an undesired vibration causing occupant discomfort or exceeding tolerable antenna inclinations. Thus, the assessment of such structures loaded by the wind should be carried out in order to secure their proper functionality and sufficient fatigue life. The wind loading includes a strong random fluctuating component with respect to time and space. Therefore the dynamic component of the wind speed can be considered as a stationary random process in individual points of a structure. Description of the static as well as dynamic components was given by many authors, e.g. [1, 2]. In particular, the problem of structure excitation by fluctuating wind component has been discussed during previous decades, see e.g. [3, 4, 5, 6] and many others. A linear approach of fluctuating component of the wind speed and along wind dynamic pressure component was presented in [7, 8]. As a consequence of the random character of the wind load, the structure response dynamic component has a random character as well.

Important role modifying the response character plays a damping and its detailed configuration. Commonly used assumption of proportional damping is satisfactory in many cases providing easier mathematical modelling. However, this assumption can be far from the reality, as for instance when a structure is equipped with tuned mass dampers. This fact being taken into consideration results in an interaction of conventional eigen-forms in the meaning of the structure itself (on the level of a deterministic problem). Moreover the random character of the response generates another source of interaction of eigen-forms.

This paper presents an innovative method for the solution of the wind response of an slender structure equipped with dynamic absorber. Only along wind random vibrations due to the effect of wind pressure on windward side are assumed. An application of the theoretical method is subsequently demonstrated on an example of a real structure.

\section{THEORETICAL ANALYSIS}

\subsection{Mathematical model}

The vibration of a straight beam with static axial force can be described by the following equation:

$$
\left.\left(E J(x) u^{\prime \prime} x, t\right)\right)^{\prime \prime}+\left(N(x) u^{\prime}(x, t)\right)^{\prime}+2 b(x) \dot{u}(x, t)+\mu(x) \ddot{u}(x, t)=p(x, t)
$$

The coefficients $E J(x), N(x), b(x), \mu(x)$ in Eq. (1) are positive time independent continuous functions $x$ with finite number of discontinuities; $p(x, t)$ is an external excitation, which can be written as a product of independent space and time components $\varphi(x) \cdot p(t)$. The structure must be kinematically stable with respect to boundary conditions to assure that the unique solution can be obtained.

Concentrating on the result of a more complicated reasoning (respecting coefficients and excitation character), it can be shown, that the solution of the Eq. (1) can be written in a form of the convergent series with respect to generalized coordinates:

$$
u(x, t)=\sum_{j=1}^{n} u_{j}(x) \cdot f_{j}\left(\theta_{j} t\right) ; \quad n \longrightarrow \infty
$$

where $u_{j}(x), \theta_{j}$ - eigen-functions, or eigen-values of the operator $\mathbf{L}\{\cdot\}$. They are given by 
the equation:

$$
\mathbf{L}\left\{u_{j}(x)\right\}+\theta_{j}^{2} \mu(x) \cdot u_{j}(x)=0 ; \quad \mathbf{L}\{u(x)\} \equiv\left(E J(x) u^{\prime \prime}(x)\right)^{\prime \prime}+\left(N(x) u^{\prime}(x)\right)^{\prime}
$$

with boundary conditions:

$$
\begin{array}{r}
u_{j}(0)=u_{j}^{\prime}(0)=u_{j}^{\prime \prime}(0)=0 \\
u_{j}^{\prime \prime}(l)=0 ; \quad\left(E J(x) u_{j}^{\prime \prime}(x)\right)^{\prime}+\left.N(x) u_{j}^{\prime}(x)\right|_{x=l}=0
\end{array}
$$

Existence of a discrete spectrum of eigen-values $\theta_{j}$ follows from positive definite operator $\mathbf{L}\{\cdot\}$. Vector $\mathbf{f}(t)=\left[f_{1}(t), . ., f_{n}(t)\right]^{T}$ conforms to a system:

$$
\begin{array}{r}
\Theta^{2} \cdot \mathbf{f}(t)+2 \mathbf{B} \cdot \dot{\mathbf{f}}(t)+\mathbf{I} \cdot \ddot{\mathbf{f}}(t)=\mathbf{p}(t) \\
\Theta^{2}=\operatorname{diag}\left[\theta_{1}^{2}, . ., \theta_{n}^{2}\right] ; \mathbf{B}=\left[\int_{S} b(x) u_{k o}(x) u_{j o}(x) \mathbf{d} x\right] ; \mathbf{p}(t)=\left[\int_{S} p(x, t) u_{k o}(x) \mathrm{d} x\right]
\end{array}
$$

where $u_{j o}(x)$ are normalized eigen-functions in following meaning:

$$
\mathbf{u}_{o}(x)=\left[u_{j o}(x)\right]=\left[u_{j}(x) / \sqrt{M_{j}}\right] ; \quad M_{j}=\int_{S} \mu(x) u_{j}^{2}(x) \mathrm{d} x
$$

System (5) should be rewritten in the normal form:

$$
\begin{aligned}
& \dot{\mathbf{F}}(t)=\mathbf{Q} \cdot \mathbf{F}(t)+\mathbf{P}(t) \\
& \mathbf{F}(t)=\left[\begin{array}{l}
\mathbf{f}_{1}(t) \\
\mathbf{f}_{2}(t)
\end{array}\right] ; \begin{array}{l}
\mathbf{f}_{1}(t)=\mathbf{f}(t) \\
\mathbf{f}_{2}(t)=\mathbf{f}(t)
\end{array} ; \mathbf{Q}=\left[\begin{array}{ll}
0 & ; \mathbf{I} \\
-\Theta^{2} & ; \quad-2 \mathbf{B}
\end{array}\right] ; \mathbf{P}(t)=\mathbf{H} \mathbf{p}(t) ; \mathbf{H}=\left[\begin{array}{l}
0 \\
\mathbf{I}
\end{array}\right]
\end{aligned}
$$

where $\mathbf{p}(t)$ - vector of $n$ independent random processes acting in generalized coordinates $u_{j o}(x)$.

A response of the continuous system is now described in generalized coordinates being given by orthonormal eigen-functions $u_{j o}(x)$ of the operator (34). Eigen functions $u_{j o}(x)$ as well as eigen-values $\theta_{k}$ are real. They represent eigen-functions and values of the non-damped system.

Functions $u_{j 0}(x)$ or $u_{j}(x)$ are orthogonal due to the symmetry of the operator $(34)$, they are continuous and make a complete functional base. The operator $\mathbf{L}\{\cdot\}$ together with $\mu(x)$ is similar to the operator of the original equation (1). It can be shown, that the system of eigenfunctions $u_{j 0}(x)$ of the operator $\mathbf{L}\{\cdot\}$ is suitable to be used as a Galerkins base for a variational analysis of the Eq. (1) as they comply not only with main but also with natural boundary conditions. Application of such a functional base excludes in advance many problems of numerical instability. Absolute convergence in individual points can be achieved for $n \rightarrow \infty$ instead of a convergence in mean which is non-satisfactory from the viewpoint of an engineering application. More detailed properties of a convergence depend on a degree of similarity of operators. However, this analysis would represent a theme for a special study.

The Eq. (5) reveals that the non-trivial interaction of $u_{j o}(x)$ or $u_{j}(x)$, when dealing with eigen-forms of the original system (1) or when investigating a system response to external excitation, comes out from a non-proportional damping which is represented by the full matrix B. The decay velocity of extra-diagonal elements of this matrix can serve for estimation how far a non-proportionality can be neglected in a deterministic case. Let's notice that the matrix $\mathbf{B}$ degenerates into the diagonal one for proportional damping. In such a case the system (5) represents $n$ independent equations and generalized coordinates $u_{j o}(x)$ became eigen-forms. On the other hand do not forget that another source of interaction of $u_{j o}(x)$ or of eigen-forms coming out of their stochastic interaction exists. 


\subsection{Random response analysis by means of spectral decomposition}

Output and input processes are continuous. It can be shown, that they enable to be written in a form being based on spectral differentials of input processes:

$$
\mathbf{p}(t)=\int_{-\infty}^{\infty} \exp (\mathrm{i} \omega t) \mathrm{d} \boldsymbol{\Phi}(\omega) ; \quad \mathbf{F}(t)=\int_{-\infty}^{\infty} \mathbf{F}^{*}(\omega, t) \mathrm{d} \boldsymbol{\Phi}(\omega)
$$

$\mathrm{d} \Phi(\omega)$ - vector ( $n$ elements) of spectral differentials of processes $\mathbf{p}(t) ; \mathrm{d} \Phi_{j}(\omega)$ are white noise type processes of variable $\omega$; their correlation is given by Wiener-Khinchin relation:

$$
\mathbf{E}\left\{\mathrm{d} \boldsymbol{\Phi}(\omega) \cdot \overline{\mathrm{d} \boldsymbol{\Phi}^{T}\left(\omega^{\prime}\right)}\right\}=\delta\left(\omega^{\prime}-\omega\right) \mathbf{S}_{p}(\omega) \mathrm{d} \omega \mathrm{d} \omega^{\prime}
$$

$\mathbf{E}\{\cdot\}$ - operator of mathematical mean value with respect to Gaussian PDF;

$\mathbf{F}^{*}(\omega, t)$ - matrix $(2 n \times n)$; unknown deterministic functions describing transformation of a random excitation in generalized coordinates into components of the random response in time;

$\mathbf{S}_{p}(\omega)$ - matrix $(n \times n)$; spectral and cross-spectral densities of processes $\mathbf{p}(t)$.

Let us introduce expressions (9) into Eq. (7). The relation obtained shell be multiplied by the vector $\overline{\mathrm{d} \boldsymbol{\Phi}^{T}(\omega)}$ from the right and then the result subjected to application of the mathematical mean operator. Properties of input processes and spectral differentials enable to interchange the application order of mathematical mean and integration operators. This manipulation gives:

$$
\int_{-\infty}^{\infty}\left(\dot{\mathbf{F}}^{*}(\omega, t)+\mathbf{Q} \cdot \mathbf{F}^{*}(\omega, t)\right) \cdot \mathbf{S}_{p}(\omega) \mathrm{d} \omega=\int_{-\infty}^{\infty} 2 \mathbf{H} \cdot \mathbf{S}_{p}(\omega) \exp (\mathrm{i} \omega t) \mathrm{d} \omega
$$

Relation (10) must be fulfilled for every $t$. It is possible, taking into account that the matrix $\mathbf{S}_{p}(\omega)$ is positive definite, only if it holds:

$$
\dot{\mathbf{F}}^{*}(\omega, t)=-\mathbf{Q} \cdot \mathbf{F}^{*}(\omega, t)+2 \mathbf{H} \cdot \exp (\mathrm{i} \omega t) ;\left.\quad \mathbf{F}^{*}(\omega, t)\right|_{t=0}=0
$$

The solution of Eq. (11) can be outlined in a form of the Laplace transform $(t \rightarrow \xi)$. Following a supposition that the matrix $(\mathbf{Q}+\mathbf{I} \cdot \xi)$ is regular, its inverse matrix should exist. If the matrix $\mathrm{Q}$ has only simple eigen-values, the respective expression can be modified in a form of the summation:

$$
\mathbf{F}_{L}^{*}(\omega, \xi)=(\mathbf{Q}+\mathbf{I} \cdot \xi)^{-1} \frac{2 \mathbf{H}}{\xi-\mathrm{i} \omega}=\sum_{k=1}^{2 n} \frac{2 \mathbf{Z}_{k} \mathbf{H}}{\mathrm{i} \omega-\xi_{k}}\left(\frac{1}{\xi-\mathrm{i} \omega}-\frac{1}{\xi-\xi_{k}}\right)
$$

$\mathbf{Z}_{k}$ - $k$-th component matrix $(2 n \times 2 n)$; if eigen-values of $\mathbf{Q}$ are simple only, the matrix $\mathbf{Z}_{k}$ has a form of a diadic product: $\mathbf{Z}_{k}=\mathbf{q}_{k(R)} \cdot \overline{\mathbf{q}_{k(L)}^{T}}$, see e.g. [9];

$\mathbf{q}_{k(R, L)}-k$-th normalized right resp. left eigen-vector ( $2 n$ elements) of the matrix Q;

$\xi_{k}-k$-th eigen-value of the matrix $\mathrm{Q}$; real part of all eigen-values is negative, therefore the system is stable; absolute values $\left|\xi_{k}\right|$ are close to $\left|\theta_{k}\right|$ for low damping values. 
Using inverse Laplace transform, one obtains a solution of the Eq. (11):

$$
\mathbf{F}^{*}(\omega, t)=\sum_{k=1}^{2 n} \frac{2 \mathbf{Z}_{k} \mathbf{H}}{\mathrm{i} \omega-\xi_{k}}\left(\exp (\mathrm{i} \omega t)-\exp \left(\xi_{k} t\right)\right)
$$

where the part with $\exp (\mathrm{i} \omega t)$ represents the stationary solution and the latter one the transition effect. Response correlation matrix $\mathbf{K}_{F}\left(t_{1}, t_{2}\right)(2 n \times 2 n)$ can be obtained using its definition:

$$
\begin{aligned}
\mathbf{K}_{F}\left(t_{1}, t_{2}\right) & =\mathbf{E}\left\{\mathbf{F}\left(t_{1}\right) \overline{\mathbf{F}^{T}\left(t_{2}\right)}\right\}=\mathbf{E}\left\{\int_{-\infty}^{\infty} \mathbf{F}^{*}\left(\omega_{1}, t_{1}\right) \mathrm{d} \boldsymbol{\Phi}\left(\omega_{1}\right) \int_{-\infty}^{\infty} \overline{\mathrm{d} \boldsymbol{\Phi}^{T}\left(\omega_{2}\right) \mathbf{F}^{* T}\left(\omega_{2}, t_{2}\right)}\right\} \\
\mathbf{K}_{F}(t) & =4 \int_{-\infty}^{\infty} \sum_{k, l=1}^{2 n} \exp (\mathrm{i} \omega t) \frac{\mathbf{Z}_{k} \mathbf{H} \mathbf{S}_{p}(\omega) \overline{\mathbf{H}^{T} \mathbf{Z}_{l}^{T}}}{\left(\mathrm{i} \omega-\xi_{k}\right)\left(-\mathrm{i} \omega-\overline{\xi_{l}}\right)} \mathrm{d} \omega \quad\left(t=t_{1}-t_{2}\right)
\end{aligned}
$$

For evaluation of the response variance matrix, the value $t=0$ is to be inserted into Eq. (14). Then the exponential factor in fact disappears.

Let's recall expressions (2), (6), (7). With respect to Gaussian character of the response its random part is fully described by correlation function:

$$
K_{u}\left(x_{1}, x_{2}, t_{1}, t_{2}\right)=\mathbf{E}\left\{u\left(x_{1}, t_{1}\right) \overline{u\left(x_{2}, t_{2}\right)}\right\}=\mathbf{u}_{o}^{T}\left(x_{1}\right) \mathbf{K}_{f 11}\left(t_{1}, t_{2}\right) \overline{\mathbf{u}_{o}\left(x_{2}\right)}
$$

where $\mathbf{K}_{f 11}\left(t_{1}, t_{2}\right)$ is a cross-correlation matrix $(n \times n)$ of time components $\mathbf{f}_{1}(t)=\left[f_{i}\left(\theta_{i} t\right)\right]$, appertaining to eigen-functions $u_{i o}(x)$. It can be stated that $\mathbf{K}_{f 11}\left(t_{1}, t_{2}\right)$ is the upper-left part of the matrix $\mathbf{K}_{F}\left(t_{1}, t_{2}\right)$ :

$$
\mathbf{K}_{F}\left(t_{1}, t_{2}\right)=\left|\begin{array}{ll}
\mathbf{K}_{f 11}\left(t_{1}, t_{2}\right) ; & \mathbf{K}_{f 12}\left(t_{1}, t_{2}\right) \\
\mathbf{K}_{f 21}\left(t_{1}, t_{2}\right) ; & \mathbf{K}_{f 22}\left(t_{1}, t_{2}\right)
\end{array}\right|
$$

In stationary case the cross-correlation function of the response is given by the form:

$$
K_{u}\left(x_{1}, x_{2}, t\right)=\mathbf{u}_{o}^{T}\left(x_{1}\right) \mathbf{K}_{f 11}(t) \overline{\mathbf{u}_{o}\left(x_{2}\right)}
$$

The expression (17) transforms the cross-correlations of the response components described in generalized coordinates $\mathbf{u}_{o}(x)$ to the scalar correlation function in length coordinate of the structure.

The Eq. (14) are very widely applicable. The matrix $\mathbf{F}^{*}(\omega, t)$ can be obtained not only by means of analytical procedure. A numerical or experimental method can be used as well. Thus the way of subsequent evaluation of outlined integrals with respect to $\omega$ is predetermined. The integration can be brought up to closed formulae for simpler cases of excitation spectral densities. Such a case occurs when spectral densities $\mathbf{S}_{p}(\omega)$ have the form of a pure rational fraction and thus the integration can be fulfilled using residuum theorem. In order to evaluate above integrals various analytical and numerical procedures can be combined. Let's append that the integrals in (14) can be understood in the Stieltjes meaning. It makes possible to proceed also various combinations of continuous spectra and excessive components with given frequency and amplitude. 


\subsection{Effects of the wind velocity fluctuation}

As a background for evaluation of the matrix $\mathbf{K}_{f}(t)$ serves the matrix of spectral densities $\mathbf{S}_{p}(\omega)$ representing external random excitation. It can be derived using the expression:

$$
\mathbf{K}_{p}(t)=\mathbf{E}\left\{\mathbf{p}(\tau) \overline{\mathbf{p}^{T}(\tau+t)}\right\}=\mathbf{E}\left\{\int_{S} \mathbf{u}_{o}\left(x_{1}\right) p\left(x_{1}, t\right) \mathrm{d} x_{1} \int_{S} \overline{\mathbf{u}_{o}^{T}\left(x_{2}\right) p\left(x_{2}, t+\tau\right)} \mathbf{d} x_{2}\right\}
$$

Making use linear character of integration and mathematical mean operations, we can write after some manipulation:

$$
\begin{array}{r}
\mathbf{K}_{p}(t)=\int_{S} \int_{S} \mathbf{u}_{o}\left(x_{1}\right) \overline{\mathbf{u}_{o}^{T}\left(x_{2}\right)} \mathbf{E}\left\{p\left(x_{1}, \tau\right) \overline{p\left(x_{2}, \tau+t\right)}\right\} \mathrm{d} x_{1} \mathrm{~d} x_{2}= \\
=\int_{S} \int_{S} \mathbf{u}_{o}\left(x_{1}\right) \overline{\mathbf{u}_{o}^{T}\left(x_{2}\right)} K_{p x}\left(x_{1}, x_{2}, t\right) \mathrm{d} x_{1} \mathrm{~d} x_{2}
\end{array}
$$

where we denoted: $K_{p x}\left(x_{1}, x_{2}, \tau\right)=\mathbf{E}\left\{p\left(x_{1}, t\right) \overline{p\left(x_{2}, t+\tau\right)}\right\}$, i.e. cross-correlation of fluctuation part of pressure in points $x_{1}, x_{2}$ being dependent on a time difference $\tau$. Fourier transform of Eq. (19) gives:

$$
\mathbf{S}_{p}(\omega)=\int_{S} \int_{S} \mathbf{u}_{o}\left(x_{1}\right) \overline{\mathbf{u}_{o}^{T}\left(x_{2}\right)} S_{p x}\left(x_{1}, x_{2}, \omega\right) \mathrm{d} x_{1} \mathrm{~d} x_{2}
$$

Spectral density function $S_{p x}\left(x_{1}, x_{2}, \omega\right)$ characterizes structure of pressure fluctuation following from air-streaming fluctuation. It consists of Davenport spectral density of wind speed fluctuation, space cross-correlation relations and geometry of the structure.

$$
S_{p x}\left(x_{1}, x_{2}, \omega\right)=c_{D}^{2} \rho^{2} v_{s}\left(x_{1}\right) v_{s}\left(x_{2}\right) S\left(x_{1}\right) S\left(x_{2}\right) 4 K \frac{v_{s, 10} \Omega^{2}}{\omega\left(1+\Omega^{2}\right)^{4 / 3}} e^{\frac{\omega}{2 \pi v_{s, 10}}} \sqrt{C_{x}\left(x_{2}-x_{1}\right)^{2}}
$$

where $\Omega=1200 \omega /\left(2 \pi v_{s, 10}\right) ; \mathrm{K}$ is the roughness coefficient; $C_{x}$ is decay coefficients; $c_{D}$ is drag coefficient; $\rho$ is air density; $v_{s}\left(x_{i}\right)$ is mean value of the wind speed in point $x_{i} ; v_{s, 10}$ is mean value of the wind speed in ten meters above the ground; $S\left(x_{i}\right)$ is area corresponding to point $x_{i}$.

\section{NUMERICAL SIMULATIONS}

The proposed theoretical method was used for the solution of the response of a linear discrete numerical model of cantilever structure with relatively closely spaced natural frequencies. It represents an existing TV tower which is $190 \mathrm{~m}$ high and is fully clamped into subsoil. The first four natural frequencies of the tower $f_{i, t}$ are given in round brackets in Figure 1 . The tower consists of the lower part made of reinforced concrete followed by steel cylinders of variable cross-sections. The top of the tower creates a laminate extension. A pendulum absorber supplemented with viscous dampers was installed into a top of the structure to suppress excessive vibrations induced by vortex-shedding. The frequency tuning $\left(f_{d}=0.92 \mathrm{~Hz}\right)$ of the 1 tone absorber was designed to be the most effective at vibrations related with the second and the third eigen-modes. The mass of the absorber represents $1 / 12$ and $1 / 10$ of generalized masses of the respective eigen-modes.

A two dimensional discrete numerical model was created in CALFEM (MATLAB FEM toolbox). The model without absorber was built using beam elements and consisted of 20 nodes. Each node had 3 degrees of freedom: the horizontal and vertical displacements and a 


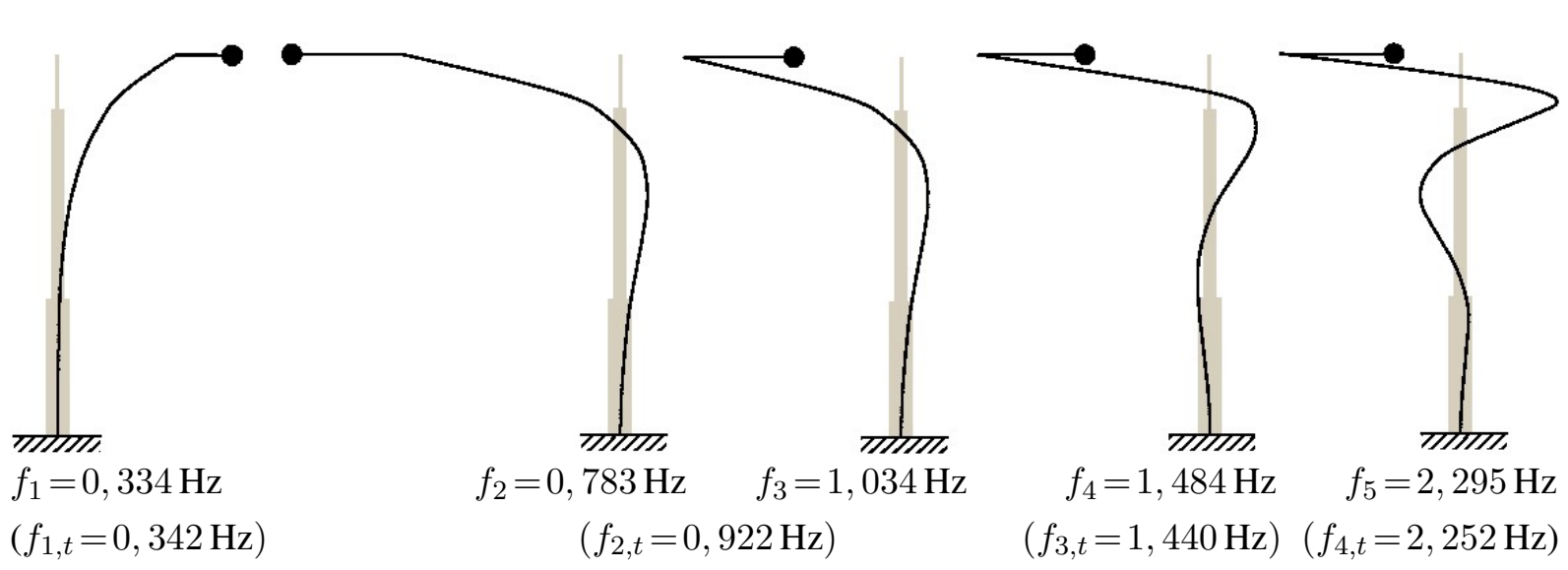

Figure 1: The first five eigen-modes of the tower with the absorber; eigen-frequencies of the tower with and without the absorber $\left(f_{i}\right.$ and $\left.f_{i, t}\right)$ are below the corresponding eigen-modes.

rotation around a horizontal axis. The absorber was subsequently modelled as a concentrated mass connected to the top of the tower with Kelvin-Voigt model having one degree of freedom in the horizontal direction. The damping matrix of the tower itself was set to be proportional to the stiffness matrix. The multiplicative coefficient related to stiffness matrix was calculated from the expected low structural damping ratio $(\zeta=0,005)$ for the first natural frequency $f_{1, t}$.

The applicability of the proposed method was examined for a set of various damping ratios of the absorber $\left(\zeta_{d}=0 \div 0.8\right)$ while the damping matrix of the tower was kept constant. The cross-correlation response matrix (17) and corresponding root mean square values (RMS) of the displacement were calculated for each damping ratio of the absorber. The numerical simulations were carried out using different number of real eigen-modes of undamped system in order to cover the influence of their mechanical interaction.

The selected results from the numerical analysis are shown in Figures 2, 3 and 4 . The RMS displacement corresponding to the structure without absorber is depicted as a function of height in Figure 2. The RMS values are shown for three different cases of real eigen-modes, that were used in the calculations. In particular, the RMS values related to vibration in the first four eigenmodes, related to vibration only in the first eigen-mode and related to vibration corresponding to the second to fourth eigen-modes are presented. It is obvious, that the vibration of the structure in the first eigen-mode represents a dominant part of the whole response.

The effectiveness of the installation of the absorber into the structure on the decrease in the dynamic response is shown in Figures 3 and 4 . In Figure 3 the RMS values are depicted for zero damping ratio of the absorber, while in Figure 4 for its optimum damping. The influence and efficiency of optimum damping ratio on the vibration related to the second to fourth eigen-mode are evident from a comparison of right graphs of both Figures 3 and 4 . It must be noted, that the influence of the absorber on the vibration in the first eigen-mode is also apparent, even though the absorber is tuned primarily to the second and third eigen-values. 

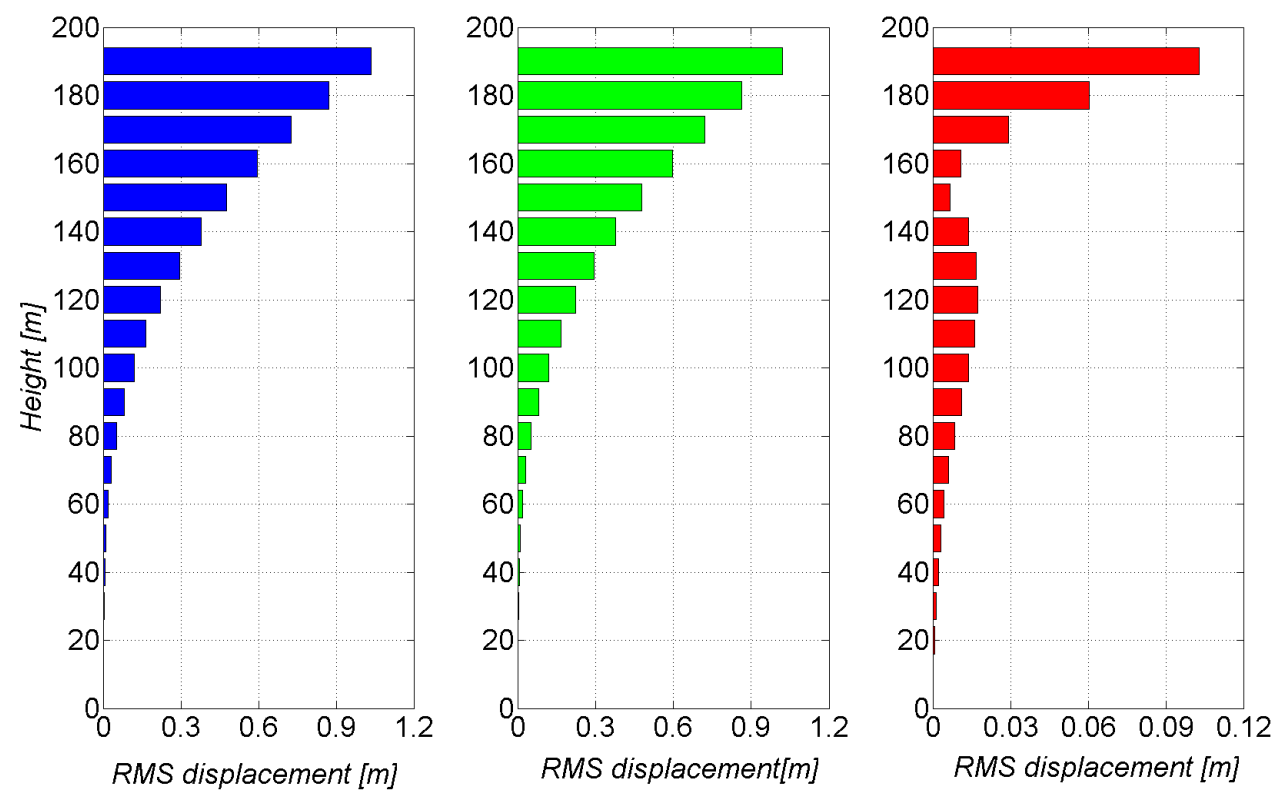

Figure 2: The RMS displacement of the tower without the absorber as a function of used real eigen-modes (left the first four eigen-modes, middle - the first eigen-mode, right - the second, third and fourth eigen-modes).
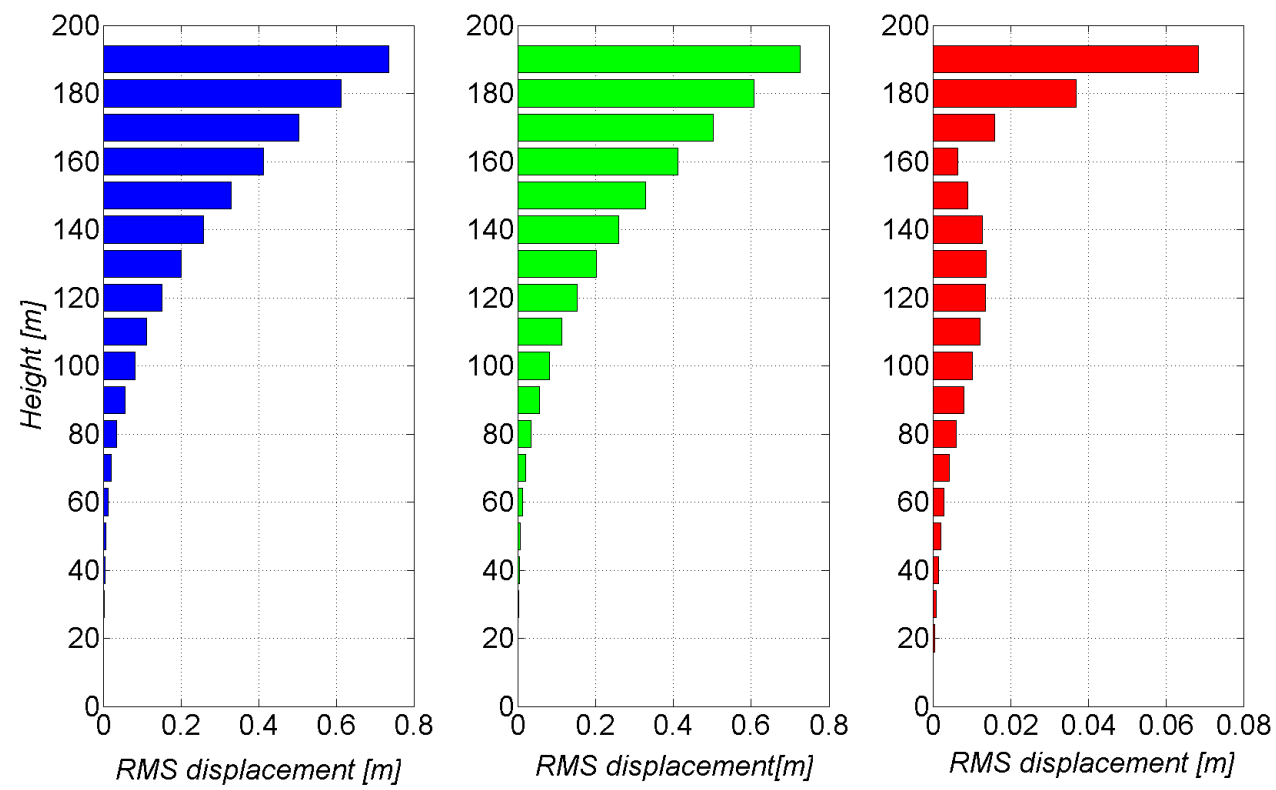

Figure 3: The RMS displacement of the tower with the absorber having zero internal damping $\left(\zeta_{d}=0\right)$ as a function of used real eigen-modes (left - the first four eigen-modes, middle - the first eigen-mode, right - the second, third and fourth eigen-modes). 

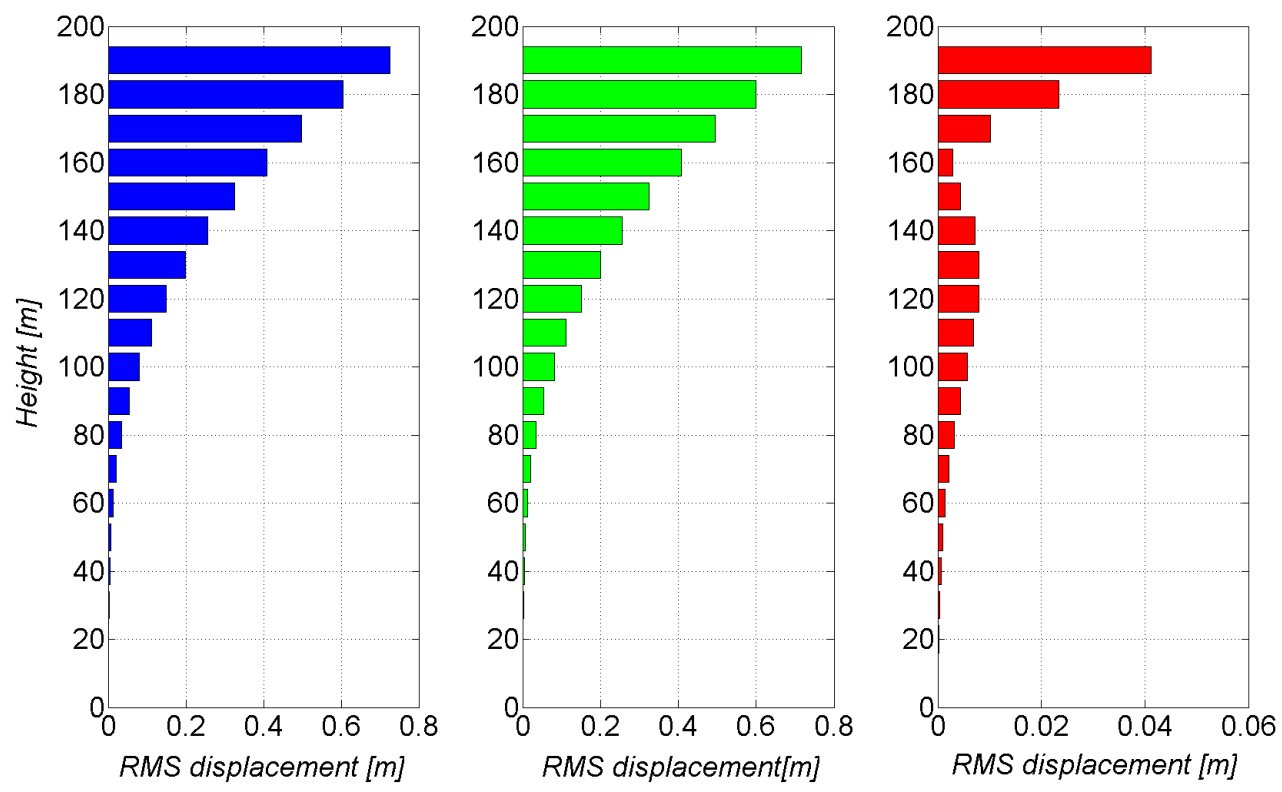

Figure 4: The RMS displacement of the tower with the absorber having optimum damping $\left(\zeta_{d}=0.18\right)$ as a function of used real eigen-modes (left - the first four eigen-modes, middle - the first eigen-mode, right - the second, third and fourth eigen-modes).

\section{CONCLUSIONS}

The paper presents an analytical method for the solution of the dynamic response of a slender structure due to a random component of the wind velocity. The mathematical model of a vertical structure being based on one-dimensional continuous elements enables to abandon a number of limiting prerequisites contradicting a reality. As the most important can be taken: (i) nonproportional damping of a structure with passive or active damping equipment; (ii) stochastic interaction of eigen-modes (vectors) which decays exponentially with increasing difference of their order; (iii) space correlation of excitation and response, etc. On the other hand a number of heuristic hypotheses have been confirmed: (i)(nearly) Gaussian character of the response; (ii) acceptability of a proportional model of a structure without any damper even in the case when it consists of materials with strongly different internal damping. The influence of initial conditions is short and decays exponentially with increasing time passed away from beginning of the excitation.

A numerical example, based on the properties of the real tower has shown the complexity of the dynamic behaviour of structure with non-proportional damping. The interaction of the eigen-modes and changes in eigen-values are demonstrated. An advantage of the model with continuous elements consists in a fact that the analysis goes in generalized coordinates which are close to eigen-forms of an investigated structure. The energy volume of individual eigenforms in general is decreasing with an increase of their order. It implies a possibility to limit easily the number of eigen-forms needed, which leads to a significant reduction of CPU time especially when analysis of random response should be done. Results are more transparent and easily to be applied in practice. Convergence is very fast and of higher type than that following from conventional approaches. 


\section{ACKNOWLEDGEMENTS}

The kind support of the Czech Science Foundation project No. 15-01035S and of the RVO 68378297 institutional support are gratefully acknowledged.

\section{REFERENCES}

[1] V. Koloušek, M. Pirner, O. Fischer and J. Náprstek, Wind Effects on Civil Engineering Structures. Academia - Elsevier, Praha, 1983.

[2] E. Simiu and R.H. Scanlan, Wind Effects on Structures. Fundamentals and Applications to Design. J. Wiley, New York, 1996.

[3] A.G. Davenport, Gust loading factor. Jour. Struct. Div. ASCE, 93, 11-34, 1967.

[4] O. Fischer and M. Pirner, Dynamics of Guyed Masts (in Czech). Academia, Praha, 1987.

[5] C. Dyrbye and S.O. Hansen, Wind loads on structures. Wiley and Sons, Chichester, 1996.

[6] A.L. Materazzi and I. Venanzi, Wind response of cable stayed masts, Proc. The Fourth European \& African Conference on Wind Engineering, Prague, 2005.

[7] M. Grigoriu and S.T. Ariaratnam, Response of linear systems to polynomials of Gaussian processes. Jour. Applied Mechanics ASME, 55, 905-910, 1988.

[8] G. Muscolino: Linear systems excited by polynomial forms of non-Gaussian filtered processes. Probabilistic Engineering Mechanics, 10, 35-44, 1995.

[9] P. Lancaster, Theory of Matrices. Academic Press, New York - London, 1969. 